\title{
SCHUBERT, Ernst, Fahrendes Volk im Mittelalter
}

\section{Joseph Morsel}

\section{OpenEdition \\ Journals}

Édition électronique

URL : http://journals.openedition.org/ifha/1305

DOI : 10.4000/ifha. 1305

ISSN : 2198-8943

Éditeur

IFRA - Institut franco-allemand (sciences historiques et sociales)

\section{Référence électronique}

Joseph Morsel, «SCHUBERT, Ernst, Fahrendes Volk im Mittelalter », Revue de l'IFHA [En ligne], Date de recension, mis en ligne le 01 janvier 2000, consulté le 22 septembre 2020. URL : http:// journals.openedition.org/ifha/1305; DOI : https://doi.org/10.4000/ifha.1305

Ce document a été généré automatiquement le 22 septembre 2020.

(CIFHA 


\title{
SCHUBERT, Ernst, Fahrendes Volk im Mittelalter
}

\author{
Joseph Morsel
}

Bien que titulaire de la chaire de Landesgeschichte de l'université de Göttingen, E.S. ne cesse de s'intéresser à des thèmes généraux et/ou transversaux, qu'il s'agisse $d u$ pouvoir royal, du pouvoir princier ou, comme ici, des «vagants », qu'il suit du Moyen Âge central aux débuts de l'époque moderne, d'un bout à l'autre de l'Empire et parfois même au-delà. Cette population mobile constitue une réalité très présente dans la société médiévale étudiée, que nous avons en général tendance à assimiler implicitement et de manière irréfléchie à des notions comme "instabilité ", " vagabondage » et finalement " marginalité ». C'est précisément à la remise en cause de ce sens commun actuel que se consacre E.S. dans cet ouvrage, où il montre que les " vagants » ne sont en aucun cas des marginaux au Moyen Âge mais que leur exclusion et leur marginalisation commencent au XVIe s.

2 Les « vagants " ne constituent pas une catégorie sociale particulière : leur seul point commun est leur mobilité, mais une mobilité liée à des fonctions particulières dont dérivent l'utilité et l'intégration sociales de ces "vagants». E.S. commence donc son étude par l'examen de cette mobilité, des fonctions sociales qui lui sont liées et des taxinomies y afférentes. Il envisage ainsi d'abord la mobilité en tant que phénomène social (ses causes, ses manifestations, ses conditions pratiques, sa géographie, les préjugés à son encontre, l'organisation collective de ses membres), puis examine un à un, dans une deuxième partie, divers types de "vagants " : le musicien et chanteur, le poète et orateur, l'artiste de son corps (danseur équilibriste, jongleur, dompteur, etc.), le clerc et écolier gyrovague, le pèlerin et le charlatan, les satellites et inverses du chevalier errant : prostituées et ribauds, enfin les vendeurs ambulants de produits ou de services plus ou moins médicaux. Cette deuxième partie fait ainsi résonner la première, plus globale et phénoménologique, ce qui l'empêche de n'être qu'un kaléidoscope d'images exotiques et met en lumière les traits saillants signalés de manière globale. On retiendra notamment le rejet d'une image immobile de la société médiévale, le rôle de la mobilité spatiale dans la réalisation de certains besoins sociaux 
(distraction, information, distribution, ostentation, etc.), l'absence de discrimination sociale ou juridique systématique, malgré l'existence d'une diffamation religieuse puis aussi juridique (les « vagants » ne sont pas hors-la-loi au sens propre du terme, mais soumis à un traitement juridique inférieur qui tient au fait qu'ils n'ont pas de protecteur attitré) : si l'on se méfie d'eux, en raison de comportements parfois délictueux, il n'y a cependant pas encore de discrimination à leur égard.

3 C'est à l'apparition de celle-ci que se consacre la troisième partie de l'ouvrage. Discrimination et criminalisation des "vagants " commencent au XVIe s., et ils disparaissent (sous leur forme médiévale) en même temps que l'ordre social médiéval. Ils se trouvent en effet à la croisée de plusieurs phénomènes radicalement contradictoires avec l'idéal d'encadrement social mis en forme par la législation de police de l'État moderne : ils sont mobiles alors que la société moderne doit être sédentaire ; ils sont sans maîtres (herrenlos) alors que les sédentaires de la nouvelle société doivent être des sujets ; ils ont un gagne-pain alors que les sédentaires assujettis doivent avoir un travail : on les assimile alors à des oisifs - l'oisiveté étant elle-même désormais un comportement anti-social. Les «vagants » de tout poil deviennent par conséquent les prototypes de l'oisif instable et incontrôlé incompatible avec le nouvel ordre social qui se met en place à l'échelle de l'Europe au XVIe s. Mais cette marginalisation ne se produit pas seulement au niveau du discours étatique : elle se retrouve également - et logiquement - au niveau de l'organisation sociale, dont l'évolution tend à faire disparaître tout interstice susceptible d'être occupé par des " marginaux » : l'intérêt de l'État est monnayé en intérêts individuels qui en assurent la réalisation. La polarisation croissante de la société sur les villes (qui contraint les villageois à s'y rendre eux-mêmes), la spécialisation, professionalisation et définition corporatiste de l'artisanat, le développement de formes non vocales de communication (avec l'imprimerie), l'institutionnalisation de l'enseignement éliminent toute nécessité des services des "vagants ", ce que montre E.S. en reprenant les exemples des divers groupes étudiés antérieurement.

4 Ce double processus d'enrégimentement et d'auto-discipline n'a pas vraiment abouti à la disparition (toujours annoncée) ou à l'encadrement complets des « vagants » - même si leur marge de manœuvre s'est extraordinairement réduite - mais avant tout à un changement à long terme des perceptions et des attitudes sociales vis-à-vis des " vagants » et " gens du voyage ", de la part des sédentaires encadrés, si bien qu'ils ont aussi servi de repoussoirs pour l'établissement et le renforcement du contrôle d'État, qui instaure frontières, barrières, passeports (au XVIIe s.) et, comme l'avait signalé E.S. lors du colloque de la Société des Historiens Médiévistes de l'Enseignement Supérieur à Göttingen en 1999 sur "L'étranger au Moyen Âge " (dont les actes viennent de paraître), la définition moderne de «l'étranger ».

5 Les « vagants » constituent ainsi les marqueurs d'une profonde évolution sociale qu'E.S. voit cristallisée dans le passeport et qu'il synthétise ainsi : « une société où la mobilité est latente se transforme en une sédentaire. Réduction et canalisation de la mobilité sont l'élément de l'auto-disciplinement (Selbstdisziplinierung) qui est mis en place dès l'époque moderne. " La démonstration d'E.S. est tout à fait pertinente, mais on peut s'interroger sur sa chronologie : qui ne perçoit que la mobilité des masses reste encore, dans les pays déjà « enrégimentés ", un enjeu (l'ordre absolu ne règnera-t-il pas le jour où chacun restera chez soi et travaillera à partir de chez soi, par ordinateur... ?) ? Et surtout, on peut se demander dans quelle mesure E.S. n'a pas tendance à idéaliser le 
Moyen Âge, caractérisé par une fondamentale liberté de mouvement, face à une Europe moderne où, un peu comme la prison de Foucault (qui n'apparaît pourtant pas dans la bibliographie), la violence symbolique d'État est construite dans les têtes en même temps que dans les institutions.

Or, l'effort de fixation des populations au sol n'a pas été créé par l'État moderne, il est en germe depuis longtemps et animé par l'Église (dont E.S. signale bien la tentative de diabolisation des histrions à partir de l'époque carolingienne, mais qu'il considère comme un échec parce que la législation civile n'a pas suivi) : il suffit de rappeler l'« encellulement » mis à jour par R. Fossier (sur l'ensemble de ces problèmes de fixation au sol par l'Église, on pourra consulter la mise au point synthétique d'A. Guerreau, « Le sens des lieux dans l'Occident médiéval : structure et dynamique d'un (espace > spécifique " in : E. Castelnuove, G. Sergi (dir.), Arti e Storia, à paraître). La condamnation à partir de l'époque carolingienne ne peut par conséquent être considérée comme un échec, elle n'est guère que le « coup d'envoi » d'un processus que les pouvoirs laïcs ont achevé pour leur compte - c'est-à-dire pour le compte de l'Église (dont ils recevaient l'essentiel de leur légitimité) ! L'opposition entre Moyen Âge et époque moderne apparaît ainsi, dans le cas du contrôle de la mobilité comme dans bien d'autres cas, très largement artificielle, et le cas des " vagants " vient bien plutôt conforter les historiens qui, à la suite de J. Le Goff ou d'A. Guerreau, plaident pour un très long Moyen Âge.

7 Joseph MORSEL 